\title{
MODEL OF THE STRUCTURED CONTINUUM, AND THE RELATION BETWEEN SPECIFIC SURFACE AREA, POROSITY AND PERMEABILITY
}

\author{
Boris Sibiryakov, Lourenildo W.B. Leite and Wildney W.S. Vieira
}

\begin{abstract}
Contrary to the Cauchy and Poisson classical seismic theory of the continuum, the new theory for structured (also called porous, fractured, or blocked) media must contain several degrees of freedom. This fact is evident because elementary blocks (grains) may transfer the motion by contact interaction, by rotation, and by group of particles. Therefore, the energy content is not only contained within the first spatial derivatives (strains), but the potential energy content is within the second (curvatures) and other higher order spatial derivatives. Thus, the equation of motion of porous media should contain higher order spatial derivatives, and may even contain infinite order spatial derivatives.
\end{abstract}

Keywords: seismic structured media, porous media, fractured media.

RESUMO. Contrariamente aos problemas clássicos da teoria sísmica do contínuo de Cauchy e Poisson, a nova teoria para meios estruturados (também denominados de porosos, fraturados e blocados) deve conter vários graus de liberdade. Este fato é evidente porque blocos elementares (grãos) podem transferir movimento por interação de contato, por rotação, e por grupo de partículas. Desta forma, o conteúdo de energia não está apenas contido nas primeiras derivadas espaciais (deformações), como também o conteúdo de energia potencial está presente na segunda (curvaturas), e em derivadas espaciais de maior ordem. Sendo assim, a equação de movimento para meios porosos deveria conter derivadas espaciais de ordem mais altas, e até mesmo uma ordem infinita de derivadas espaciais.

Palavras-chave: meios sísmicos estruturados, meios porosos, meios fraturados. 


\section{INTRODUCTION}

The present paper is part of a major project aiming to predict the stresses and strains using $P$ and $S$ wave velocities to localize small pressure areas in oil and gas productive layers as natural suction pumps. The project is devided into different relative independent parts.

The first part, for stress prediction, is related to conventional seismic investigations to obtain information on the $\mathrm{P}$ and $\mathrm{S}$ wave velocities, and also the configuration of seismic boundaries.

The second part will predict the stress and strain in the geological structures using the information obtained in the first part. Additionally, the nontrivial behavior of pressure will be predicted, because pressure can increase and decrease in depth, and create what we call natural pumps that is the mechanism to push fluids towards the low pressure zones. Predicting these natural pumps is a major aim of this project.

The third element of stress prediction is related to predicting the discontinuity in pressure between solids and fluids, which depends on pore space structure; the present study falls within this third piece.

To begin predicting the stress and strain for real geological structures, we need to know the $P$ and $S$ velocities and the seismic boundary configuration, which is a separate classical seismic problem. In the present description, we focus on isotropic models, but the equations are more complicated for anisotropic situations.

The data acquired must contain three components. From land-observed data, we can use $S$ waves from horizontal vibroseis with VSP technology. From marine observed data, we can use AV0 technology to look for converted P-S-P waves. In certain cases, we can use petrophysical measurements of borehole data.

The first discussions on the topic of pore space and integral geometry were presented by Sibiryakov (2002) and Sibiryakov \& Prilous (2007). The theory of porous media is based on integral geometry because this mathematical discipline addresses collective geometrical properties of real collectors (reservoirs). Santalo (1953) showed that these collective properties correspond to porosity, specific surface area (SSA) parameter, average curvature and Gaussian curvature. For example, porous and cracked media generally have a small porosity, but very large SSA parameter, which creates anomalous high $\gamma=v_{S} / v_{P}$ ratios, and which results in negative Poisson coefficient, $\sigma=\frac{1-2 \gamma^{2}}{2-2 \gamma^{2}}$.

The popular tubular pore space model has very high crosssectional curvature, $K_{1}$, and very small longitudinal curvature, $K_{2}$. Therefore, the Gaussian curvature $K_{G}=\sqrt{K_{1} K_{2}}$ is much less than average curvature $K_{\mathrm{A}}=\frac{K_{1}+K_{2}}{2}$. In granular media, $K_{\mathrm{G}}$ and $K_{\mathrm{A}}$ are similar (Smirnov, 1964).

\section{METHODOLOGY}

\section{Equation of motion for structured media}

The new structured continuum model contains an internal geometry of a micro-inhomo geneous medium described mainly by porosity and the SSA parameter. By structured continuum model, we mean a media formed by grains and/or by blocks, limited by cracks and/or porous containing fluids (gas, water, oil), as depicted in Figures 1 and 2.

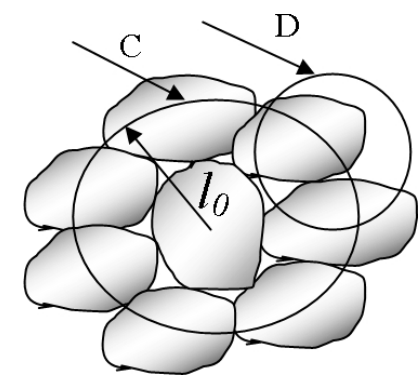

Figure 1 - Element of the structured medium, where $l_{0}$ is an average distance between grains or porous. The problem is to create an equilibrium equation for an arbitrary element of the discrete medium. An equation of equilibrium exists for the surface $C$, but does not for the surface $D$

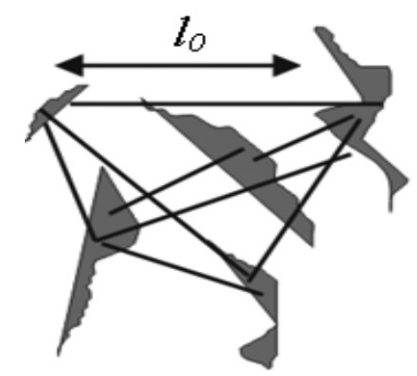

Figure 2 - Another complex element of the structured medium, where $l_{0}$ is an average distance between grains or cracks.

The porosity, $f$, is described as a fraction of the empty volume space, $V_{E}$, to the total volume, $V_{T}$, of the material including the solid and empty space: $f=\frac{v_{E}}{v_{T}}$. The empty space may contain gas and liquid.

The SSA parameter is the ratio between the real surface of pores (and cracks) and the volume of the specimen, $\sigma_{0}=$ $\frac{S}{V_{T}}\left[\mathrm{~cm}^{-1}\right]$, and this quantity is used to solve petrophysical and chemical problems; the SSA is measured from mercury $(\mathrm{Hg})$, and from gas absorption methods. Examples of geometrical figures, for instance, from a tetrahedron to a sphere provide decaying values of the form $\sigma_{0} \propto \frac{1}{a}$, where $a$ is the solid parameter, such as radius or length of a regular side (Mavko et al., 1999).

Figure 1 shows a volume element of a structured body, where $l_{0}$ is the average distance between grains (or the distance between pores). A theorem of Integral Geometry relates the SSA 
parameter $\sigma_{0}$ to $l_{0}$ by the formula, Sibiryakov (2002),

$$
\sigma_{0} l_{0}=4(1-f) .
$$

This same Eq. (1) applies to Figure 2 that presents a different situation, where a volume element of a structured body is characterized by cracks, and also by the SSA parameter $\sigma_{0}$, and by the arithmetic average distance, $l_{0}$, between the grains (or the distance between the cracks). In grainular media, we have negative curvature for the grains, but positive curvature for the pore space. In cracked media, we generally find zero curvature for the boundary porous/solid. Positive solid curvature for cavernous pores gives a large pressure jump between solid and liquid (Landau \& Lifschitz, 1961).

In Equation (1), $f$ is the porosity; therefore, given a sample with the SSA $\sigma_{0}$, there is automatically an average size $l_{0}$ for the microstructure. The distinction between the classical and structured continuum should be clear in Figures 1 and 2. In the volume bounded by the surface $C$, there is an equation of equilibrium because all forces cancel, while there is no equation of equilibrium in the volume bounded by surface $D$ because all forces are concentrated on one side of the grain, and the other side contains no forces.

The objective here is to create a new spatial model for wave propagation in structured media characterized by porosity, permeability, and specific suraface area.

We consider some finite body volume, where surface forces are applied on a sphere of radius $l_{0}$, while the inertial forces are applied in the structure center. It is not possible to make an elementary volume approach zero, making the points of the surface to coincide to the point in the center, and the inertial forces are similar to the classical continuum; we must consider a finite volume as a representative body volume, and the inertial forces at different positions on the surface.

The main feature of this approach is to fill all space, including pores and cracks, with a force field. This approach provides a continuous image of a real, complicated medium. The natural laws must apply to continuous image of the medium, and not to the real image. The one-dimensional space operator for field translation from point $x$ to point $x \pm l_{0}$ is given by the symbolic equation, Maslov (1973),

$$
u\left(x \pm l_{0}\right)=u(x) e^{ \pm l_{0} D_{x}} .
$$

This form applies to any field, and here $u(x, y, z, t)$ stands for particle displacement, and

$$
\dot{u}(x, y, z, t)=\frac{d}{d t} u(x, y, z, t),
$$

for particle velocity.
From formula (2), the one-dimensional field translation operator, $D_{x}=\frac{\partial}{\partial x}$, can be rewritten for 3D space as follows, Sibiryakov \& Prilous (2007),

$$
\begin{aligned}
P\left(D_{x}, D_{y}, D_{z}\right) & =\frac{\sinh \left(l_{0} \sqrt{\Delta}\right)}{l_{0} \sqrt{\Delta}} \\
& =E+\frac{l_{0}^{2}}{3 !} \Delta+\frac{l_{0}^{4}}{5 !} \Delta \Delta+\ldots,
\end{aligned}
$$

where $E$ is the unit operator, $\Delta$ is the Laplace operator, and

$$
\frac{\partial^{2}}{\partial x^{2}}+\frac{\partial^{2}}{\partial y^{2}}+\frac{\partial^{2}}{\partial z^{2}}=\Delta \text {. }
$$

The operator $P$ is also represented by the special symbolic averaging operator given by:

$$
\begin{gathered}
P\left(D_{x}, D_{y}, D_{z}\right)=\frac{1}{4 \pi} \int_{0}^{2 \pi} \\
\int_{0}^{\pi} e^{l_{0}\left(D_{x} \sin \theta \cos \phi+D_{y} \sin \theta \sin \phi+D_{z} \cos \theta\right)} \sin \theta d \theta d \phi .
\end{gathered}
$$

In a classical continuum, we apply the impulse conservation law, $F_{i}=m \ddot{u}_{i}$, to any element of the medium, or under the form

$$
F_{i}=\frac{\partial \sigma_{i k}}{\partial x_{k}}=\rho \frac{\partial^{2}}{\partial t^{2}} u_{i},
$$

where $m$ stands for mass, and $\rho$ for density.

In the present case, we need to fill all pore space by a force field, and we write the infinite order equation of motion as:

$$
\begin{gathered}
P\left(\frac{\partial \sigma_{i k}}{\partial x_{k}}\right)=\left(E+\frac{l_{0}^{2}}{3 !} \Delta+\frac{l_{0}^{4}}{5 !} \Delta \Delta+\cdots\right) \frac{\partial \sigma_{i k}}{\partial x_{k}} \\
=\rho \frac{\partial^{2}}{\partial t^{2}} u_{i} .
\end{gathered}
$$

Instead of real stress, which may change from a large value (in the grain) down to zero (in the pore space), we use the image of real stress. Namely, we use a continuous field constructed by applying the $P$ operator to the real complicated force field. For this continuous image of real stress, $P\left(\sigma_{i k}\right)$, we can apply the conservation law of physical impulse. In a classical model of the continuum, the operation $P=E$ comes from nature itself. The constructed model (5) of the continuum requires some mathematical operations to create the continuum medium.

Considering the one-dimensional case for simplicity and extracting results considering plane waves, stationary motion, $u(x, y, z, t) \rightarrow u\left(x, \omega=k_{B} v_{B} ; l_{0}\right)$, we have a simpler infinite order equation of motion in the form, Sibiryakov \& Prilous (2007),

$$
\begin{aligned}
(E+ & \left.\frac{l_{0}^{2}}{3 !} \frac{\partial^{2}}{\partial x^{2}}+\frac{l_{0}^{4}}{5 !} \frac{\partial^{4}}{\partial x^{4}}+\ldots\right) u_{x x} \\
& +k_{B}^{2} u\left(x, k_{B} ; l_{0}\right)=0,
\end{aligned}
$$


where $k_{B}=\omega / v_{B}$ stands for both $\mathrm{P}$ and $\mathrm{S}$ waves. Considering only one term, we write Eq. (6) as:

$$
u_{x x}+k_{B}^{2} u=0 .
$$

Considering only two terms, we write Eq. (6) as:

$$
u_{x x}+\frac{l_{0}^{2}}{3 !} u_{x x x x}+k_{B}^{2} u=0 .
$$

We can examine a solution of the equation of motion (6) in the stationary form,

$$
u(x, y, z, t)=U(x, y, z) e^{i \omega t} .
$$

For one-dimensional case, $U(x, y, z)$ has the form $U(x)$, and:

$$
U(x)=A(k) e^{i k x}=A\left(\frac{\omega}{v}\right) e^{i \frac{\omega}{v} x} .
$$

We are not applying the Fourier transform, but are examining discrete values of the temporal radial frequency $(\omega)$, and of the wavenumbers $\left(k_{x}, k_{y}, k_{z}\right)$.

\section{Condition for a negative Poisson coefficient}

Substituting representation (10) into Eq. (6), we obtain the dispersion equation for the unknown wavelength $k=\frac{2 \pi}{\lambda}$,

$$
\frac{\sin \left(k l_{0}\right)}{k l_{0}}=\left(\frac{k_{B}}{k}\right)^{2}
$$

where $k_{B}=\frac{\omega}{v_{B}}(v$ is velocity and $\omega=2 \pi f)$ is the wavenumber of the usual $\mathrm{P}$ or $\mathrm{S}$ waves. Eq. (11) is a transcendental equation with respect to the unknown $k$. For the condition $l_{0} \rightarrow 0$, $k \rightarrow k_{B}$, we have the usual wave velocities infinite small structures. In the case that $l_{0}$ is not very small, then $k<k_{B}$, the dispersion velocity $v=\frac{\omega}{k}$ is greater than $v_{B}$, and the $v_{P}$ and $v_{S}$ velocities are decreasing due to structure.

Numerically examining Eq. (11) shows that the P-wave velocity decreases more rapidly than the S-wave velocity. Therefore, the ratio $\gamma=\frac{v_{S}}{v_{P}}$ may be greater than $\frac{1}{\sqrt{2}}$. For the classical continuum model,

$$
\gamma=\sqrt{\frac{\mu}{\lambda+2 \mu}}
$$

and if $\lambda=0$, the

$$
\gamma=\sqrt{\frac{1}{2}} \approx 0.705,
$$

where $(\lambda, \mu)$ are the Lamè parameters. Now, if we measure $\gamma>0.705$, we must have $\lambda<0$. As a result, the Poisson coefficient $\sigma=\frac{1}{2} \frac{\lambda}{\lambda+\mu}$ is negative because $\lambda<0$, and for small values the denominator is positive. Experimental observations of this strange result were first published by Gregory (1976). The negative Poisson coefficient is due to a dispersion phenomenon in structured media. The real Poisson coefficient measured in statics, instead of wave propagation, does not produce such a strange result.

Figure 3 shows the relation between the $\mathrm{P}$ and $\mathrm{S}$ wavenumbers, $\frac{k_{B}(\omega)}{k_{B}(0)}$, versus the ratio $\varepsilon=2 \pi \frac{l_{0}}{\lambda_{B}}$. It is clear that the $P$ wavenumber (curve 1 ) increases faster than the $S$ wavenumber (curve 3), which means that the P-wave velocity decreases faster than the S-wave velocity. The ratio $\gamma=\frac{v_{S}}{v_{p}}$ increases from 1 to 1.25 , from low to high frequencies.

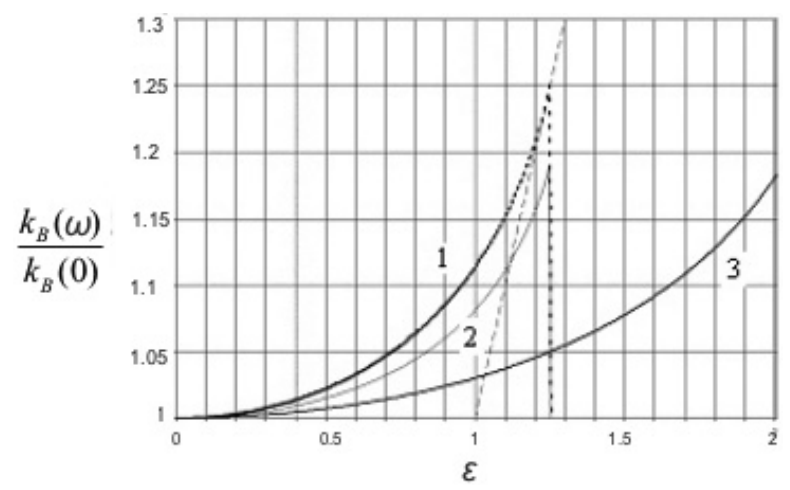

Figure 3 - Plot of Eq. (11), real roots. The horizontal axis gives $\varepsilon=2 \pi\left(l_{0}\right) /$ $\left(\lambda_{B}\right)$ ratio. Curve 1 shows the increasing wavenumber ratio $k_{P}(\omega) / k_{p}(0)$; therefore, $v_{P}$ decreases by increasing frequency. Curve 3 shows the same for $\mathrm{S}$ waves, $k_{s}(\omega) / k_{s}(0)$, where $v_{S}$ decreases with increasing frequency. Curve 2 shows the increase of $\gamma=v_{S} / v_{P}$ ratio up to a negative Poisson coefficient $\sigma$

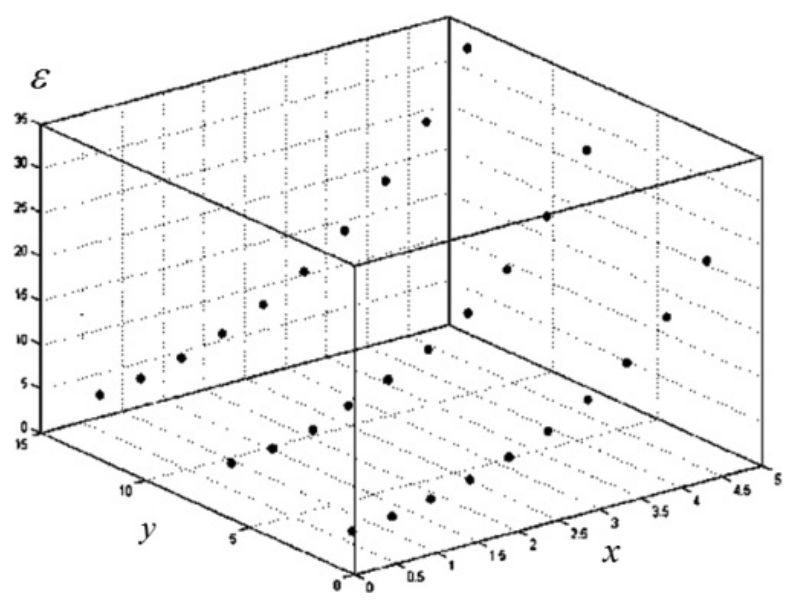

Figure 4 - Plot of Eq. (11), complex roots. The horizontal coordinates are $x=\operatorname{Real}\left(k l_{0}\right)$ and $y=\operatorname{Imag}\left(k l_{0}\right)$. The vertical coordinate is $\varepsilon(x, y)=$ $k_{B} l_{0}$. If $k_{B} l_{0} \ll 1$, only real roots exist.

Figure 4 shows the real and imaginary parts of the roots of the dispersion Eq. (11) as a function of $\varepsilon=2 \pi \frac{l_{0}}{\lambda_{B}}$. The roots of Eq. (11) are obtained for $k l_{0}=n \pi,(n$ integer), and 
very large $k$ with a velocity that is very small. The interpretation of this figure is that if $\varepsilon \ll x$, then there is a wave with abnormally small velocities less than $v_{S}$. Additionally, velocities are discrete in porous media, while velocities are continuous in classic media. This situation is analogous in quantum mechanics (the discrete points represent a discrete spectrum of eigenvalues).

Clearly, Eq. (6) contains derivatives of infinite order, and this circumstance is due to the several degrees of freedom for structured bodies. For $l_{0} \rightarrow 0$, we have the usual equations of motion for a classical continuum space model.

\section{The long wave approach. Equations of motion as Korteweg-de-Vries and Boussinesq types}

For small $l_{0}$ values compared to wavelength, it is possible to reduce the equation of motion (6) of an infinite order to an equation of the fourth order by neglecting the terms containing values of order $l_{0}^{4}$ and above. In this case, we can consider some nonlinear relations between stress and strain.

Figure 5 depicts a general case of linear and nonlinear stressstrain relation, and Savarensky (1975) and White (1983), among other authors, present more general cases of the stress-strain nonlinear behavior in solids. Sibiryakov (1974) describes the two solutions for one-dimensional nonlinear equation of motion. Figure 5 does not show the stress and strain complexities of the loading and unloading process; therefore the area of the hysteresis loop representing dissipative energy is not present.

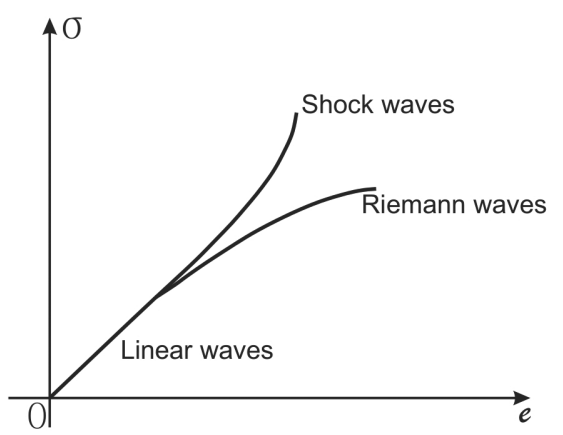

Figure 5 - Qualitative relation between stress $(\sigma)$ and strain $\left(e=u_{x}\right)$ showing the domains of linear elastic waves, and nonlinear Shock and Riemann waves.

For the nonlinear case, the reduction of stress can take place by the increase in deformation for rocks and subsurface. Therefore, shock waves are absent in such a media, and the nonlinear waves are the Riemann waves (Zeldovich \& Raizer, 1966).

The reduced equation of motion (6) takes the following form:

$$
\frac{\partial}{\partial x_{k}}\left(E+\frac{l_{0}^{2}}{3 !} \Delta\right) \sigma_{i k}=\rho \ddot{u}_{i} \text {. }
$$

Furthermore, we consider the $1 \mathrm{D}$ case, and use the nonlinear relation between stress and strain given by:

$$
\sigma_{x x}=(\lambda+2 \mu)\left(u_{x}-b u_{x}^{2}\right),
$$

where $b$ is a given constant. We can write the corresponding Eq. (12) in the form:

$$
u_{x x}\left(1-2 b u_{x}\right)+\frac{l_{0}^{3}}{3 !} u_{x x x x}=\frac{1}{c^{2}} \ddot{u}_{i} .
$$

By changing of variables, $\xi=c t-x$ and $\eta=c t+x$, the equation of motion (14) reduces to a type similar to the Korteweg-de-Vries, (KdV)-equation, Dazin \& Johnson (1989):

$$
u_{\eta}-2 b u u_{\xi}+\frac{l_{0}^{2}}{3 !} u_{\xi \xi \xi}=0 .
$$

The classical KdV-equation has another sign in the nonlinear term, and Eq. (15) thus has no solutions of the soliton type, and the role of the nonlinear term will be presented below. If the nonlinear term is absent, Eq. (15) is similar to the Boussinesq type, i.e.:

$$
u_{x x}+\frac{l_{0}^{3}}{3 !} u_{x x x x}=\frac{1}{c^{2}} \ddot{u}_{i},
$$

where the second term in the left is the dispersion term.

Now, we look at the solution of the Eq. (14) in the wave form given by:

$$
u(t, x)=c T F\left(\frac{t-\alpha \frac{x}{c}}{T}\right),
$$

where $T$ is a characteristic time of the pulse, $c$ is a given constant value, and $\alpha$ is greater than unity. Changing variables as $\xi=\frac{t-\alpha \frac{x}{c}}{T}$, and assuming that

$$
F^{\prime}(\xi)=\frac{\partial F(\xi)}{\partial \xi}=\varphi(\xi),
$$

we can write the ordinary nonlinear Eq. (14) in the form:

$$
\varphi^{\prime \prime}+\frac{3 !(\alpha-1)}{l_{0}^{2} \alpha^{4}} \varphi=-\frac{3 ! 2 b}{l_{0}^{2} \alpha} \varphi^{2} .
$$

To analyze Eq. (18), consider that the following quantities can be valid:

$\frac{3 !\left(\alpha^{2}-1\right)(c T)^{2}}{l_{0}^{2} \alpha^{4}}=1, \quad \delta=\frac{l_{0}}{c T}, \quad \alpha=1+\frac{1}{2} \delta^{2}$.

Therefore, the value of $\varphi(\xi)$ is represented by the product $\varphi=$ $\varphi_{0} \bar{\varphi}$, where the constant $\varphi_{0}$ is equal to the characteristic value of strain; an example value, is the elastic limit of shear deformation. With the assumptions considered above, a simpler equation 
is obtained for the variable $\bar{\varphi}$. Ignoring the bar over $\bar{\varphi}$, we have a nonlinear equation for (18):

$$
\varphi^{\prime \prime}+\varphi+\beta \varphi_{0} \varphi^{2}=0,
$$

where $\beta=\frac{3 ! 2 b}{\alpha \delta^{2}}$. Therefore, in spite of $\varphi_{0}$ being very small, and $\beta$ being large, the product $\beta \varphi_{0}$ is not very small. Additionally, $\alpha \approx 1$, and $\delta$ is a very small value for the small structure size compared to the wavelength. Hence, the dispersion phenomena in micro-inhomogeneous media increase the nonlinear effects, and a form of the solution of Eq. (20) is shown in Figures 6 and 7.

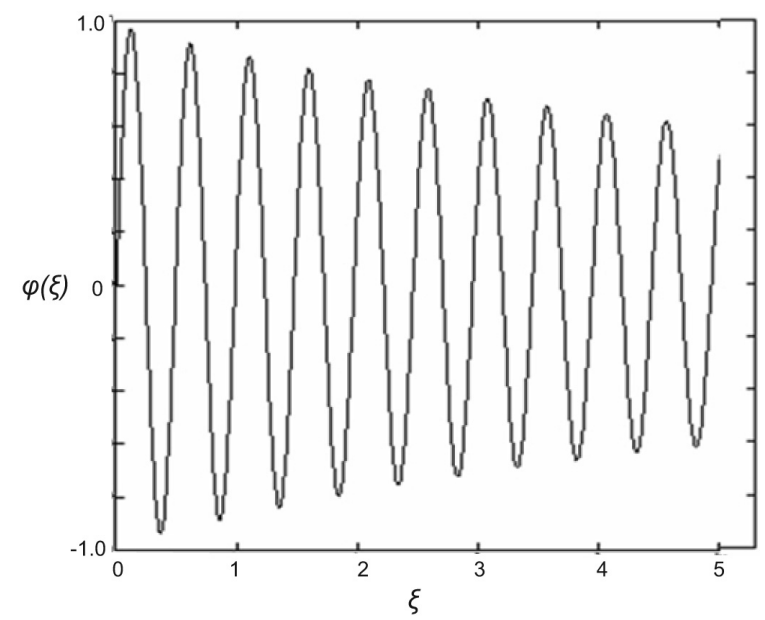

Figure $\mathbf{6}$ - Qualitative plot of the function $\varphi(\xi)$ as a result of the inverse of Eq. (21) showing the attenuation with distance of an initial normalized sinusoidal causal pulse. The horizontal axis is divided in units 1 to 10 , and the vertical axis normalized between -1.0 and +1.0 . The nonlinear parameter is $\beta \varphi_{0}=0.1$. The logarithmic decrement is nearly constant.

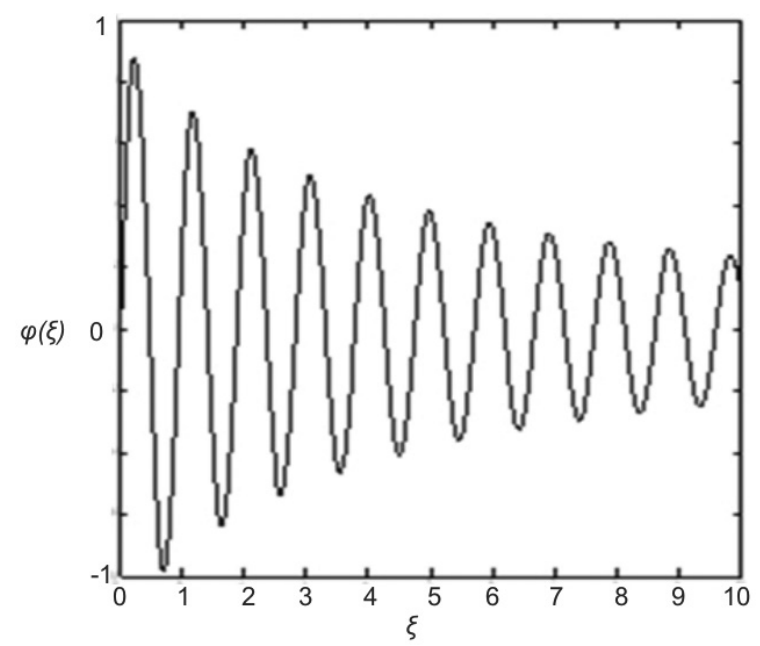

Figure 7 - Qualitative plot of the function $\varphi(\xi)$ as a result of the inverse of Eq. (21) showing the attenuation with distance of an initial normalized sinusoidal causal pulse. The horizontal axis is divided in units 1 to 10 , and the vertical axis is normalized between -1.0 and +1.0 . The nonlinear parameter is $\beta \varphi_{0}=0.5$. The variation of the logarithmic decrement is now visible.
The rigorous solution of $\mathrm{Eq}$. (20) is given by the following in implicit elliptical integral form:

$$
\xi=\frac{t-\alpha \frac{x}{c}}{T}=\int_{0}^{\varphi(\xi)} \frac{d p}{\sqrt{1-p^{2}-\beta \varphi_{0} p^{3}}} .
$$

This solution for $\beta \varphi_{0} \rightarrow 0$ tends to the usual sinusoidal function, and is written as:

$$
\begin{aligned}
& \int_{0}^{\varphi} \frac{d p}{\sqrt{1-p^{2}-\beta \varphi_{0} p^{3}}} \\
= & \frac{t-\alpha \frac{x}{c}}{T} \approx \arcsin (\varphi(\xi)),
\end{aligned}
$$

from where, for $\beta \varphi_{0}=0$,

$$
\varphi(\xi)=\sin \left(\frac{t-\alpha \frac{x}{c}}{T}\right),
$$

corresponds to a consistent sinusoidal solution.

A more common exact solution of Eq. (20) takes a form:

$$
\frac{t-\alpha \frac{x}{c}}{T}=\int_{0}^{\varphi} \frac{d p}{\sqrt{C_{1}+C p+1-p^{2}-\beta \varphi_{0} p^{3}}}
$$

where $C_{1}$ and $C$ are arbitrary constants, and the integral (24) describes a wider class of phenomena than the integral (21).

We can linearize Eq. (20) using, instead of function $\varphi^{2}$, the good approximation $\varphi^{2}=\varphi \varphi_{0}=\varphi(\xi) \cos (\xi)$, in which the $\cos (\xi)$ term is the solution of the linear equation found when $\beta \varphi_{0} \rightarrow 0$. In other words, we can write $\varphi^{2} \approx \varphi \cos (\xi)$, and $\xi=\frac{t-\alpha \frac{x}{c}}{T}$. As a result, we can linearize Eq. (20) to a form of Mathieu's equation that describes the parametric resonance of mechanical excitation and oscillation given by:

$$
\varphi^{\prime \prime}+\left(1+\frac{\varphi_{0}}{\delta^{2}} \cos (\xi)\right) \varphi=0 .
$$

This equation contains both, stable and unstable solutions. The instabilities contain attenuation and increasing vibrations as catastrophes. The role of the parameter $\varphi_{0}$ is not for its small value, but is more significant under the ratio $\frac{\varphi_{0}}{\delta^{2}}$.

The attenuation of a sinusoidal pulse with distance due to nonlinearity is shown in Figures 6 and 7. The nonlinearity parameter is not very small due to the large factor $\frac{1}{\delta^{2}}$. These figures show how the attenuation of a sinusoidal pulse should appear. When the nonlinear parameter is equal to zero, i.e., $\beta \varphi_{0}=0$, attenuation is absent; if $\beta \varphi_{0}=0.1$, a small attenuation occurs with almost constant logarithmic decrement; for larger nonlinear parameter, say $\beta \varphi_{0}=0.5$, sufficient attenuation occurs without 
of constant decrement. Eq. (25) also contains increasing solutions, but we deal only with damping vibrations in this paper.

The authors Egorov \& Mashinskii (2002) realized a laboratory experiment with an artificial sample of dry sandstone (general properties: length, $1 \mathrm{~m}$; diameter, $0.76 \mathrm{~m}$; porosity, 0.3 ; and density $2 \mathrm{~g} / \mathrm{cm}^{3}$ ) subjected to simultaneous excitation by two vibroseis with frequencies $6100 \mathrm{~Hz}$ and $7720 \mathrm{~Hz}$. The signal spectrum was observed at the round surface of the cylindrical sample, and the source was placed at the flat end of the cylinder. The result of the experiment is shown in Figure 8.

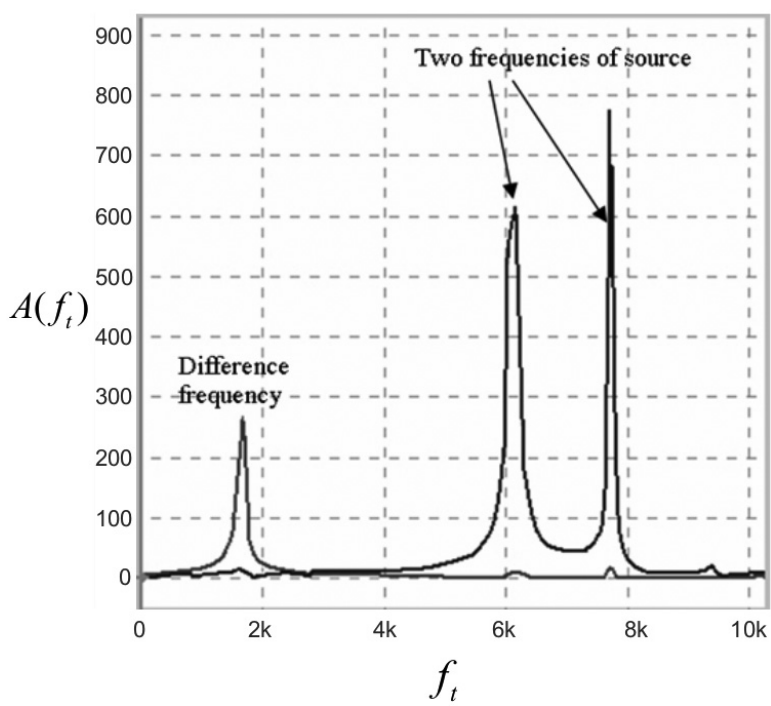

Figure 8 - Observed spectrum amplitude, $A\left(f_{t}\right)$, of quasi-sinusoidal signals for an artificial laboratory sandstone model. The horizontal axis shows the frequency $f_{t}$ from 0 to $10.000 \mathrm{~Hz}$, and the vertical axis shows the amplitude from 0 to 900 units. The data are according to Egorov \& Mashinskii (2002).

The receivers registered also the difference frequency of $1620 \mathrm{~Hz}$ at a distance of $75 \mathrm{~cm}$ from the source. It is interesting that the amplitude of the difference frequency is extremely high; i.e., it reached the order of $30 \%$ percent of initial signal (Egorov \& Mashinskii, 2002). The classical approach related to the second-order equations of motion predicts this effect, which is proportional to the square of strain. The dispersion phenomena in porous media sharply strengthen nonlinear processes, so even weak fluctuations are accompanied by appreciable nonlinear effects (Mashinskii \& Egorov, 2011).

\section{Porous media with viscous liquid}

Let us suppose that at the gravity center (i.e., at the point $\mathbf{x}=$ $(x, y, z)$ ) the particle velocity is $\dot{u}_{i}(\mathbf{x})$ (see Fig. 1). The average distance from point $\mathbf{x}$ to the grain boundary is $f l_{0}$, and the velocity on the grain boundary is therefore represented by a Taylor series in the form:

$$
\begin{gathered}
\dot{u}_{i}\left(\mathbf{x}+f l_{0}\right)=\dot{u}_{i}(\mathbf{x}) \\
+f l_{0}\left(\frac{\partial \dot{u}_{i}}{\partial x} \cos (r, x)+\frac{\partial \dot{u}_{i}}{\partial x} \cos (r, y)+\frac{\partial \dot{u}_{i}}{\partial x} \cos (r, z)\right) \\
+O\left(\left(f l_{0}\right)^{2}\right) .
\end{gathered}
$$

This expansion is bounded by the first order term with respect to $f l_{0}$, and the total sum in the right side is equal to zero. Therefore, there is no displacement between grain and viscous fluid on the boundary of the grain.

We can consider that the derivatives act on the contact skeleton-fluid with accuracy up to small values in the second order, and the center of gravity, $\mathbf{x}$, is in the pore. The relation between the radial and normal derivatives of the particle velocity, with components $(x, y, z)$, as in $\mathrm{Eq}$. (26), is given by:

$$
\frac{\partial \dot{u}_{i}}{\partial r}=\frac{\partial \dot{u}_{i}}{\partial n} \cos (r, n) .
$$

The average value of $\cos (r, n)$ in three-dimensional space is 0.5. The relation between the particle velocity and the derivative of the particle velocity is given by the following:

$$
\dot{u}_{i}=-\frac{1}{2} f l_{0} \frac{\partial \dot{u}_{i}}{\partial n}
$$

for the sum in Eq. (26) to be null.

The surface force, $F_{S_{i}}$, of viscous friction is proportional to the viscosity, $\eta$, and to the derivative of the particle velocity, $\dot{u}$, with respect to the normal, $n$, to the surface that separates matrix and fluid:

$$
F_{S_{i}}=\eta \frac{\partial \dot{u}_{i}}{\partial n}=-2 \frac{\eta}{f l_{0}} \dot{u}_{i} .
$$

The volume force of viscous friction is a product of the surface force to the SSA parameter of the pore space, i.e.:

$$
F_{i}=\sigma_{0} \eta \frac{\partial \dot{u}_{i}}{\partial n}=-2 \frac{\sigma_{0} \eta}{f l_{0}} \dot{u}_{i}=8 \frac{\eta(1-f)}{f l_{0}^{2}} \dot{u}_{i} .
$$

In formula (30), the factor $\frac{1}{2 f} \sigma_{0}^{2}$ plays the role of inverse permeability, which means that the permeability $\phi$ is a geometric parameter, and we have the equality:

$$
\phi\left(f, \sigma_{0}\right)=2 \frac{f}{\sigma_{0}^{2}} .
$$

This formula is shown in Figure 9, from which we learn that $\sigma_{0}$ diminishes the permeability very quickly.

This approach shows that it is unnecessary to use Darcy's law for wave physics or percolation (Biot, 1962).

In the structured continuum model, the forces created by internal stresses act via the $P$-operator. The Eq. (6) of motion is of infinite order, because many internal waves with different velocities exists in micro-inhomogeneous bodies. The Cauchy-Poisson 


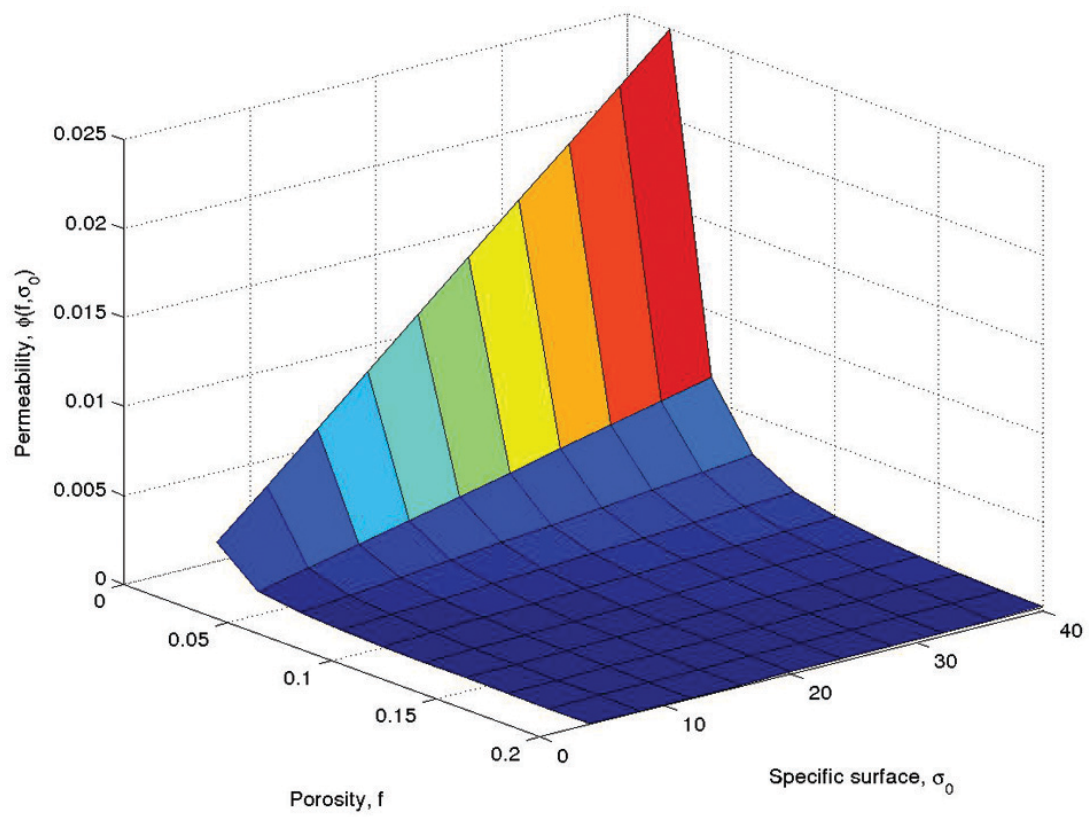

Figure 9 - Plot of Eq. (31). The horizontal axis are porosity, $f$, and the SSA parameter, $\sigma_{0}$, and the vertical axis is the permeability, $\phi=\phi\left(f, \sigma_{0}\right)$.

hypothesis is that $P=E$, which means that any property is an average for any arbitrary small volume. In reality, it is not possible to use arbitrary small volumes, because the representative volume must contain some elementary structures (grains), and they have internal motions, rotations, etc. If $l_{0} \Rightarrow 0$, we have the classical standard equation of motion, and $P=E$.

Considering one-dimensional case, in the classical continuum $(P=E)$ model we obtain the wave equation:

$$
u_{x x}=\frac{1}{c^{2}} \ddot{u} .
$$

And with viscous friction included we obtain the Telegraph equation:

$$
u_{x x}=\frac{1}{c^{2}} \ddot{u}+\frac{\beta}{f} \dot{u},
$$

where we have added the dissipative forces in the second term on the right of Eq. (32), based on the Eq. (30). Here, $\beta$ can be summarized as $\beta=\sigma_{0}^{2} \frac{\eta}{M}$, where $M=\rho c^{2}$. Therefore,

$$
\beta=\beta_{P}=\sigma_{0}^{2} \frac{\eta}{\lambda+2 \mu}\left[T L^{-2}\right],
$$

is a property of the stress relaxation time for the $\mathrm{P}$ waves, and $\beta=\beta_{S}=\sigma_{0}^{2} \frac{\eta}{\mu}$ for the $S$ waves, where we can see that $\beta_{S}$ is larger than $\beta_{P}$. The quantities $\lambda$ and $\mu$ are the Lamè parameters, and $\eta$ the viscosity.

For the solution of Eq. (33), we separate the amplitude and time components in the form:

$$
u(x, t)=U(x) e^{i \omega t} .
$$

Substituting (34) into (33), we obtain the differential equation for the amplitudes given by:

$$
U^{\prime \prime}+\left(\frac{\omega^{2}}{c^{2}}-i \frac{\beta \omega}{f}\right) U=0 .
$$

For the solution of (35) we find that:

$$
U=U_{0} e^{m x}, \quad \text { and } \quad m= \pm \sqrt{\frac{\omega^{2}}{c^{2}}-i \frac{\beta \omega}{f}} .
$$

Therefore, Eq. (34) has now the form:

$$
u(x, t)=U_{0} e^{i \omega\left(t \pm \frac{x}{c} \sqrt{1-i \frac{c^{2} \beta}{f \omega}}\right)} .
$$

Let's analyze two conditions for binomial expansion of the square root in the exponent of (37). The first is for $\frac{\omega \beta}{f}<<1$, then:

$$
\sqrt{1-i \frac{c^{2} \beta}{f \omega}} \approx 1-i \frac{c^{2} \beta}{2 f \omega} .
$$

The attenuation is the imaginary part of the exponent and, from (37) and (38), it is given by:

$$
e^{i \omega \frac{x}{c}\left(\frac{i c^{2} \beta}{f \omega}\right)}=e^{-x \frac{c \beta}{f}},
$$


that does not depend on $\omega$ frequency. The second is for the opposite situation, $\frac{\omega \beta}{f}>>1$, where we have the attenuation in the form:

$$
e^{-x \frac{\beta \omega}{\sqrt{f \omega}}}=e^{-x \beta \sqrt{\omega}},
$$

which gives the attenuation proportional to the square root of the $\omega$ frequency.

For porous media, the viscous friction creates velocities only by the fluctuation of particles, and the friction forces therefore act by the $P-E$ operator Sibiryakov et al. (2011). The equation of motion in this porous and viscous media can be represented in the form:

$$
\begin{gathered}
P\left(\frac{\partial \sigma_{i k}}{\partial x_{k}}\right)-i \frac{\beta \omega}{2 f}(P-E) U+k_{B}^{2} U=0, \\
k_{B}=\frac{\omega}{v_{P, S}}
\end{gathered}
$$

where $U=U(x, y, z, \omega)$. The corresponding dispersion equation is given by:

$$
\frac{\sin \left(k l_{0}\right)}{k l_{0}}\left(k^{2}-i \frac{\beta \omega}{2 f}\right)+i \frac{\beta \omega}{2 f}=k_{B}^{2} .
$$

The case $l_{0} \rightarrow 0, k^{2} \rightarrow k_{B}^{2}$, means that in classical continuum media, with infinitely small structure size, the viscous friction is absent.

Continuing examining the dispersion Eq. (42), then for small values of $l_{0}$ we have the expansion:

$$
\frac{\sin \left(k l_{0}\right)}{k l_{0}}=1-\frac{\left(k l_{0}\right)^{2}}{3 !}+\cdots,
$$

and we can now write Eq. (42) as:

$$
k^{2}-i \frac{\beta \omega}{3 ! 2 f}\left(k l_{0}\right)^{2}=k_{B}^{2} .
$$

Using the relations $\sigma_{0} l_{0}=4(1-f)$ and $\beta=\sigma_{0}^{2} \frac{\eta}{\rho c^{2}}$, we can write (44) in the form:

$$
k^{2}\left(1-i \frac{4 \eta(1-f)^{2}}{3 f} \omega\right)=k_{B}^{2} .
$$

Then, we can write:

$$
k \sqrt{\left(1-i \frac{4 \eta(1-f)^{2}}{3 f} \omega\right)}=k_{B} .
$$

For small ratios $\frac{\eta \omega}{\rho c^{2}}<<1$, we have that:

$$
k\left(1-i \frac{2}{3} \frac{4 \eta(1-f)^{2}}{3 f} \omega\right) \approx k_{B} .
$$

Now, we need an approximation for the wavenumber $k$ in terms of $k_{B}$, and:

$$
k \approx \frac{k_{B}}{\left(1-i \frac{2}{3} \frac{4 \eta(1-f)^{2}}{3 f} \omega\right)},
$$

after an expansion,

$$
k \approx k_{B}\left(1+i \frac{2}{3} \frac{\eta(1-f)^{2}}{\rho c^{2} f} \omega\right),
$$

where the structure parameters $(f, \eta, \rho, c)$ and $\omega$ are present in the complex part.

\section{CONCLUSIONS}

Presently, fluid percolation theory based on Darcy's law ignores stress-strain state in solids. Additionally, this percolation theory contains porosity, but does not contain the SSA parameter that creates forces to stop percolation.

We need to predict the stress-strain in solids, and pressure discontinuity between phases; and this discontinuity depends on the structure of pore space, and not only on porosity.

It is unnecessity to use Darcy's law for determining permeability, because permeability is only a geometric property of porous medium. The permeability value is directly proportional to porosity, and is inversely proportional to the square of the SSA parameter for a specimen. In a submitted paper, Sibiryakov et al. (2013), we show that the fluid velocity $\left(\dot{u}_{0}\right)$ into a borehole is approximated by $\dot{u}_{0}=K f \eta \sigma_{0}^{-2}$, where $K$ is a constant, $f$ is porosity, $\eta$ is the viscosity, and $\sigma_{0}$ the SSA parameter. In this equation, $\eta$ is usually included into $K$, leaving $f \sigma_{0}^{-2}$ playing the role of permeability.

Porosity and the SSA parameter enable using alternative methods to measure the permeability.

The equation of motion for long wavelengths compared to the specimen structure does not result in the wave equation, but in the telegraph equation that describes the propagation and diffusion of waves.

\section{ACKNOWLEDGMENTS}

The authors would like first to thank the paper Reviewers for their patience and positive contribution for discussions, and in special about the proposed relationship between the mineral type and the SSA parameter; and also to look for a relationship for permeability with another parameter besides the SSA parameter.

We would like to thank the Brazilian institutions UFPA (Universidade Federal do Pará), Fase 5 of FINEP (Financiadora de 
Estudos e Projetos), ANP (Agência Nacional do Petróleo) and PETROBRAS (Petróleo Brasileiro S/A) for research support, and specially to the project National Institute of Science and Technology (Instituto Nacional de Ciência e tecnologia, INCT-GP, MCT/CNPq/FINEP).

Major thanks to Science Without Borders of CAPES/CNPq of the Brazilian Federal Government, which sponsors research concerning oil exploration.

\section{REFERENCES}

BIOT MA. 1962. Mechanics of deformation and acoustic propagation in porous media. Journal of Applied Physics, 23: 1492-1498.

DAZIN PG \& JOHNSONRS. 1989. Solitons: an introduction. Cambridge University Press. $226 \mathrm{pp}$.

EGOROV GV \& MASHINSKII EI. 2002. Biharmonic compressional and shear waves in the porous sample of artificial sandstone under axial pressure (in russian). Technology of Seismic Exploration, 1(1): 72-77.

GREGORY AR. 1976. Fluid saturation effect on dynamic elastic properties of sedimentary rocks. Geophysics, 41(5): 895-921.

LANDAU L \& LIFSCHITZ M. 1961. Course of theorectical physics: theory of elasticity. Nauka, Moscow. 165 pp.

MASHINSKII El \& EGOROV GV. 2011. The amplitude effects in direct wave, which propagate in cemented sandstone with pressure (in russian). Russian Geology and Geophysics, 52(5): 725-731.

MASLOV VP. 1973. Theory of operators (in russian). Nauka, Moscow. $559 \mathrm{pp}$.

MAVKO G, MUKERJIT \& DVORKIN J. 1999. The rock physics handbook. Cambridge University Press, London. 329 pp.
SANTALO L. 1953. Introduction to integral geometry. Hermann and Cia. Editors. $123 \mathrm{pp}$.

SAVARENSKYE. 1975. Seismic waves. Mir, Moscow, Russia. 349 pp.

SIBIRYAKOV BP \& PRILOUS BI. 2007. The unusual small wave velocities in structural bodies and instability of pore or cracked media by small vibration. WSEAS Transactions on Applied and Theoretical Mechanics, 2(7): 139-144.

SIBIRYAKOV BP, PRILOUS BI \& KOPEYKIN AV. 2011. The nature of instabilities in blocked media and seismological law of Gutenberg-Richter. WSEAS Transactions on Applied and Theoretical Mechanics Issue 2, 6, 69-79.

SIBIRYAKOV BP, LEITE LWB \& VIEIRA WWS. 2013. Behavior of stresses and hydrodynamics from multicomponent seismic data. Submitted to Journal of Geophysics - RBGf. 19 pp.

SIBIRYAKOV BP. 1974. The one dimensional problem of attenuation of nonlinear waves in grounds (in russian). Geology and Geophysics, 13(11): 108-115.

SIBIRYAKOV BP. 2002. Supersonic and intersonic cracking in rock-like material under remote stresses. Theoretical and Applied Fracture Mechanics, 38(3): 255-265

SMIRNOV VI. 1964. Course of higher mathematics. Pergamon Press, London. $630 \mathrm{pp}$.

WHITE JE. 1983. Underground sound. Applications of seismic waves. Elsevier, Amsterdam, Netherlands. 253 pp.

ZELDOVICH YB \& RAIZER YP. 1966. Physics of the shock waves and high temperature hydrodynamics phenomena. Nauka, Moscow, 64-66.

Recebido em 28 maio, 2013 / Aceito em 19 dezembro, 2013

Received on May 28, 2013 / Accepted on December 19, 2013

\section{NOTES ABOUT THE AUTHORS}

Boris Sibiryakov is a Special Professor of Geophysics at the Russian Academy of Sciences (RAS), Novosibirsk Branch, and at the State University of Novosibirsk. At present he is a Special Visiting Professor for the geophysics graduate program at the Federal University of Pará (UFPA), Brazil, participating in the Project Science Without Borders of CAPES/CNPq. He obtained a Masters and a Doctorate titles in Geophysics from the University of Saint Petersburg. He develops research in applied seismic theory of fractured media for oil and gas exploration.

Lourenildo W.B. Leite is a professor of Geophysics in the undergraduate and graduate programs of the Federal University of Pará (UFPA), Brazil. He obtained a Masters and a Doctorate in Geophysics from Saint Louis University, Missouri, USA. He develops research in applied seismic theory for oil and gas exploration focusing on velocity analysis, stack, inversion, migration, and attribute analysis. He is a member of the SBGF, SEG, EAGE and SSA scientific societies.

Wildney W.S. Vieira is a Doctorate student at the graduate program in Geophysics of the Federal University of Pará (UFPA), Brazil. He obtained a Bachelors in Mathematics (in 2007), and a Masters of Geophysics (in 2011), both degrees at UFPA. He develops research in inversion techniques for seismic theory applied to oil and gas exploration. His interest has been focused on imaging through a complete processing workflow using data quality control, velocity analysis, inversion and migration. He is a member of the SBGF scientific society. 